\title{
Une bibliothèque Art déco
}

La bibliothèque de Reims

\section{Matthieu Gerbault}

\section{(2) OpenEdition Journals}

Édition électronique

URL : https://journals.openedition.org/rbnu/3093

DOI : $10.4000 /$ rbnu.3093

ISSN : 2679-6104

Éditeur

Bibliothèque nationale et universitaire de Strasbourg

\section{Édition imprimée}

Date de publication : 1 mai 2010

Pagination : 16-27

ISSN : 2109-2761

Référence électronique

Matthieu Gerbault, "Une bibliothèque Art déco », La Revue de la BNU [En ligne], 1 | 2010, mis en ligne le 01 janvier 2021, consulté le 12 juin 2021. URL : http://journals.openedition.org/rbnu/3093 ; DOI https://doi.org/10.4000/rbnu.3093 Attribution - Pas d'Utilisation Commerciale - Partage dans les Mêmes Conditions 4.0 International. 


\section{Une bibliothèque Art déco : la bibliothèque de Reims}

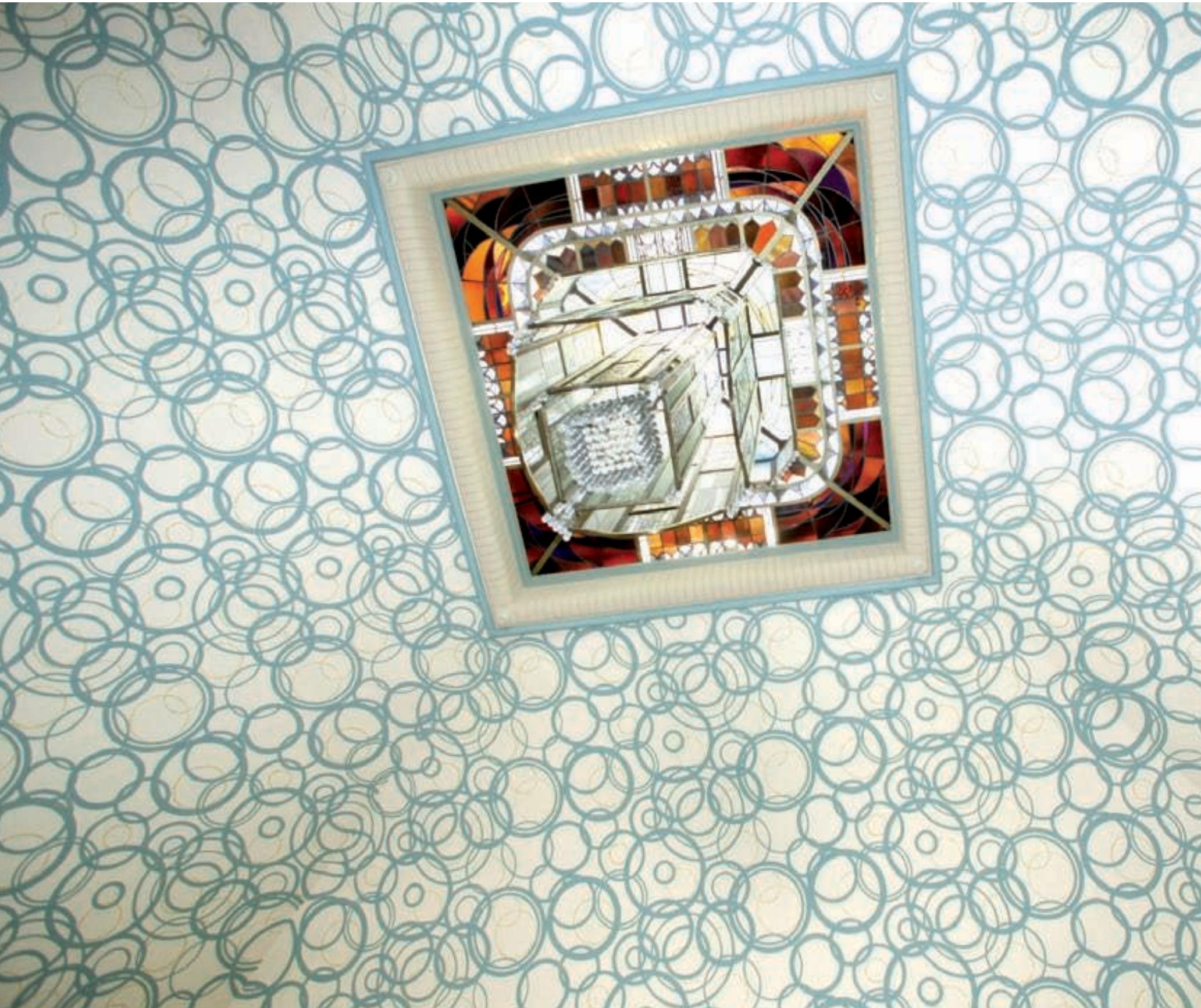




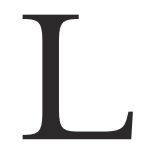

a bibliothèque Carnegie, véritable joyau de l'architecture Art déco, fait aujourd'hui la fierté de Reims. Depuis son inauguration en 1928, les brochures touristiques n'ont cessé d'attirer l'attention sur la majesté de ses dimensions et le luxe de ses décors. En même temps, le bâtiment a toujours suscité un vif intérêt chez les architectes et les bibliothécaires, intérêt relayé dans la presse spécialisée et toujours vivace aujourd'hui ${ }^{1}$. Il faut dire que cette bibliothèque a parfaitement su répondre, à l'époque de sa construction, aux besoins nouveaux de l'accueil du public et de la conservation des livres. Si ces besoins ont fortement évolué au cours du $\mathrm{XX}^{\mathrm{e}}$ siècle, justifiant la modernisation récente de la BMVR de Reims, la bibliothèque Carnegie reste aujourd'hui un modèle architectural pour la valorisation et la conservation des fonds patrimoniaux. Cet article se propose de revenir sur la conception originale du bâtiment, sa réalisation finale, sa réception chez les professionnels et le public après son inauguration.

\section{Le projet de construction}

La décision de construire une nouvelle bibliothèque à Reims a été prise au lendemain de la Grande Guerre. La ville est alors totalement dévastée : entre 1914 et 1918, les bombardements allemands n'ont épargné aucun édifice remarquable. Parmi ceux-ci, l'Hôtel de ville, où siégeait l'ancienne bibliothèque municipale depuis 1818 , a été détruit par un obus incendiaire le 3 mai 1917 - heureusement sans dommage pour les collections les plus précieuses, transférées à Paris et à Sainte-Clotilde. À la fin de la guerre, Reims n'est plus que ruines. L'ampleur du désastre suscite la compassion de l'opinion internationale et se traduit par un puissant mouvement de générosité chez des mécènes américains. C'est dans ce contexte que la Fondation Carnegie pour la paix internationale décide, en 1920, de subventionner l'édification d'une bibliothèque à Reims, « qui a si indignement souffert du bombardement allemand et qui symbolise, à ses yeux, les traditions et l'âme de la France ${ }^{2}$ ". La Fondation verse à la ville une somme importante : 200 ooo dollars (3 millions de francs).

Le terrain retenu par la municipalité pour le nouveau bâtiment est un espace triangulaire en plein centre-ville, à proximité de la cathédrale, entre la rue de l'École de médecine et la rue du Cardinal de Lorraine.

Après consultation de la Société des architectes de la Marne, c'est Max Sainsaulieu qui obtient la direction du nouveau chantier : la commande lui est confirmée par le maire en décembre $1920{ }^{3}$. Né à Péronne en 1870, formé auprès d'Edmond Duthoit puis à l'Ecole des beaux-arts de Paris, Sainsaulieu avait effectué de nombreuses réalisations à Reims et à Soissons avant la guerre. La construction d'une bibliothèque est une commande nouvelle pour cet architecte qui est plutôt spécialiste d'édifices religieux ou d'immeubles de rapport ${ }^{4}$.

Voulant réaliser un bâtiment conforme aux besoins de la lecture publique, il entreprend, dès janvier 1921, un voyage d'étude qui le conduit dans sept bibliothèques françaises et suisses assez récentes : Clermont, Lyon, Genève, Fribourg, Zurich, Bâle ; un autre voyage, en février, lui permet de découvrir Bruxelles, Louvain, Aulnaye, La Haye et enfin Leyde. 
Au cours de ces visites, il est accompagné par Henri Loriquet, le conservateur de la bibliothèque municipale, Pol Neveux, inspecteur général des bibliothèques et Charles Roche, le maire de Reims. Ces voyages sont approfondis par la lecture complémentaire d'études sur les bibliothèques américaines envoyées par le Centre européen de la dotation Carnegie 5 .

Les enseignements tirés de ces voyages nous sont connus grâce à un rapport daté du $1^{\text {er }}$ février $1921^{6}$, qui s'ouvre par une sévère critique des bibliothèques anciennes : « la très grande majorité des bibliothèques françaises sont filles de la Révolution; et, logées dans des locaux : couvents, séminaires, chapelles, hôtels de villes, etc., qui n'étaient pas faits pour elles, on les trouve toutes mal distribuées, mal équipées, mal éclairées, mal chauffées, mal ventilées, mal surveillées, mal adaptées à la conservation des livres et au service des lecteurs qui, naturellement, ne les comprennent ni ne les fréquentent 7 ". A l'inverse, les bibliothèques visitées fournissent des réponses plus appropriées aux enjeux de la lecture publique. Ainsi, celles de Clermont et de Lyon retiennent l'attention de la délégation rémoise par l'aspect pratique et hygiénique des conditions de stockage ; mais leurs salles de lecture, trop sombres et mal desservies, déçoivent. Les bibliothèques suisses suscitent plus d'enthousiasme : dotées de magasins de stockage bien éclairés, de systèmes de classement ingénieux et de catalogues pratiques sur fiches mobiles, elles offrent des salles de lecture confortables et vastes. Celle de Zurich, éclairée par des baies zénithales et latérales, apparaît même comme un modèle à suivre ${ }^{8}$.

A l'appui de ces voyages d'étude, Sainsaulieu ébauche un premier projet en 1921. Ce projet est composé de trois ensembles, conformément à la commande qui lui a été faite : une bibliothèque municipale ; un pavillon privé pour le conservateur ; un jardin clôturé. La bibliothèque, conçue pour emmagasiner 400 ooo livres, sépare de manière très rationnelle les espaces de travail pour le personnel, les espaces d'accueil du public et les espaces de stockage : le bâtiment principal, grand immeuble rectangulaire en pierres de taille blanches, est dédié à l'accueil du public (salle de lecture et salle de conférences) ainsi qu'au logement du personnel (bureaux, conciergerie). Les collections, en accès indirect, sont stockées dans de grands magasins en hémicycle sur cinq niveaux, rejetés à l'arrière du bâtiment principal et desservis par un escalier central et un monte-charge électrique. Ces grands magasins seront en briques rouges, rythmées par des chaînages de pierres de taille blanches.

L'avant-projet de Sainsaulieu, soumis au Conseil supérieur des bibliothèques le 7 avril 1921, reçoit un accueil enthousiaste : l'architecte est félicité pour ce bâtiment qui réaliserait " la meilleure installation de bibliothèque provinciale, la mieux adaptée à la conservation et à la manutention du livre, ainsi qu'aux facilités du public et du personnel ${ }^{9}$ ". L'avantprojet est approuvé par le conseil municipal le 6 juillet $1921^{10}$.

Il est pourtant remanié l'année suivante. La mairie décide, tout d'abord, de suspendre la construction du pavillon du conservateur : le devis annoncé excède la somme mise à disposition par la dotation Carnegie ${ }^{11}$. Reporté dès l'origine, ce pavillon n'a jamais pu être construit, malgré les demandes réitérées de Loriquet ${ }^{12}$. Par ailleurs, le Conseil supérieur des bâtiments civils demande de nombreuses retouches. Dans un rapport daté de décembre $1922^{13}$, après avoir salué l'aspect novateur de la bibliothèque et accepté la forme rayonnante des magasins, il est demandé les modifications suivantes : harmonisation des façades extérieures (la brique devant être abandonnée au profit de la pierre blanche) ; agrandissement des fenêtres des magasins 


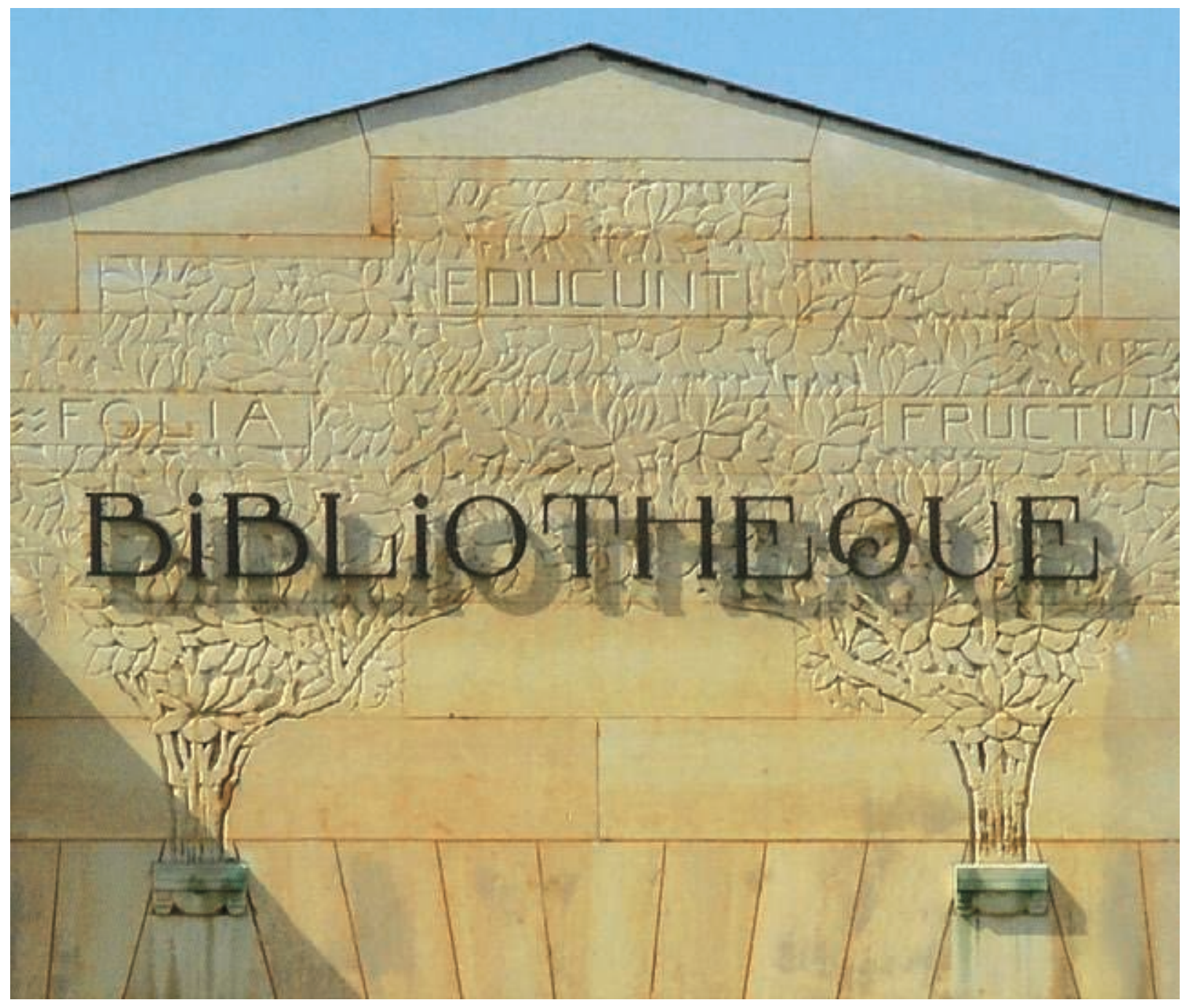


de stockage pour en améliorer l'éclairage ; augmentation de la hauteur sous plafond de certaines salles, comme le cabinet des manuscrits, et amélioration de leur accessibilité ; report des vitrages au-dessus des terrasses en toiture ; ajout d'une cour anglaise au rez-de-chaussée. Sainsaulieu accepte de bonne grâce d'harmoniser les façades et de modifier les toitures. Il se plie beaucoup plus difficilement aux autres demandes : l'agrandissement des ouvertures des magasins, notamment, lui paraît inadapté à

la conservation des livres anciens,

faits pour être protégés de la lu-

mière ${ }^{14}$; acceptant finalement

de s'exécuter, il soumet à

la mairie et au Conseil un

nouveau projet modifié le

16 mars 1923. Ce projet B

est adopté le 16 avril ${ }^{15}$.

\section{Les travaux}

et la réalisation finale

La pose solennelle de la

première pierre avait eu lieu

le 19 juillet 1921, en présence

de Nicholas M. Butler, prési-

dent de la Fondation Carnegie et

de Myron T. Herrick, ambassadeur des

Etats-Unis en France.

Les travaux débutent en 1923 pour s'achever en 1927.

D'ultimes interventions et retouches ont lieu au

cours de l'année 1928, année de l'inauguration et de l'ouverture au public.

Le bâtiment final se présente au visiteur comme une superbe réalisation, à la fois sobre dans ses formes et luxueuse dans son décor.

La façade principale, simple rectangle de pierre de taille, est percée en son centre d'un grand portique surmonté d'un fronton triangulaire, orné de motifs d'arbres en bas-relief qui sont l'œuvre du sculpteur rémois Edouard Sediey. De chaque côté de l'ouverture, on aperçoit les armes et devises des Etats-Unis et de Reims. Au sommet de la façade, une frise décorative rappelle les noms des plus illustres auteurs de la Champagne ${ }^{16}$. On accède à la bibliothèque en gravissant quelques marches jalonnées de deux grandes vasques de Sèvres ; arrivé sur le perron, décoré de mosaïques posées par Biret, le visiteur traverse une grande porte en fer forgé noir, exécutée par l'entreprise Schwartz-Haumont, aux motifs de rosaces entrelacées et rehaussées par des cabochons de cuivre doré. Le portique, reconstitué lors de l'Exposition internationale des Arts décoratifs de Paris, en 1925, y a été couronné par une médaille d'or ${ }^{17}$.

A l'intérieur, un grand vestibule en marbre accueille le lecteur. Il est égayé en son centre par une fontaine, symbole de la connaissance. Sur les parois, vingt mosaïques stylisées, dessinées par Sauvage, représentent l'ensemble des activités humaines, preuve que la bibliothèque est un lieu encyclopédique. Au plafond, une grande coupole où se déploient des cercles verts symbolisant la frondaison du savoir est éclairée en son sommet par un grand pendentif en verre, œuvre du maître-verrier rémois Jacques Simon.

Le vestibule donne accès à l'ensemble des espaces. Face à l'entrée, un guichet en marbre permet de passer commande des livres désirés. A gauche, derrière la salle du catalogue, le personnel accède au secrétariat et au bureau du conservateur. L'intention originelle de créer un cabinet des médailles a été abandonnée, permettant d'augmenter sensiblement les dimensions du bureau du conservateur.

A droite, une salle de lecture de quarante places offre une atmosphère studieuse : entièrement lambrissée d'étagères donnant accès aux usuels et à quelques collections de périodiques, elle est égayée par de grands pastels de fleurs dessinés par une artiste rémoise, Madeleine Lacourt : ces pastels, simplement décoratifs, ont été ajoutés quelques semaines avant l'inauguration du bâtiment pour atténuer le caractère un peu trop hiératique des grands lambris ${ }^{18}$.

La salle est éclairée, au plafond, par un vitrail de 


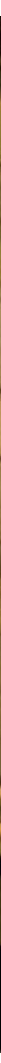


Jacques Gruber qui représente un livre ouvert sur les armes de la ville de Reims. Trois grandes baies, elles aussi réalisées par Gruber, s'ouvrent côté rue. Elles représentent des abeilles stylisées, symbolisant l'effort et le travail. La salle est exposée au nord, ce qui permet de ne pas souffrir des fortes chaleurs en été ${ }^{19}$.

L'emplacement et la luminosité de la salle de lecture étaient primordiaux pour Sainsaulieu. Dans une lettre à Jean Montariol, architecte de la future bibliothèque de Toulouse, Sainsaulieu donne le conseil suivant : « le point important autour duquel j’ai tourné pendant longtemps, c'est d'abandonner le préjugé qui consiste à mettre coûte que coûte la salle de lecture dans l'axe, comme cela existe dans la plupart des bibliothèques allemandes (plan de Strasbourg), ce qui encombre tout et vous conduit à entourer cette salle de grands bâtiments et à ne l'éclairer que par le haut ${ }^{20}$ ".

A côté de la salle de lecture, une grande salle d'exposition s'ouvre au visiteur. Dotée d'un parquet original posé par le mosaïste Noël (carrés de chêne et d'azobé fixés dans du ciment de Portland), elle est meublée de vitrines fixes en son centre. La destination finale de cette grande salle a suscité de nombreuses discussions entre le conservateur et l'architecte. Loriquet a réussi à l'imposer pour mettre en valeur des expositions temporaires, malgré l'avis défavorable de Sainsaulieu, qui n'en voyait pas l'utilité. Ce dernier pensait plutôt en faire une salle de conférences, imaginant qu'elle pourrait servir ensuite d'extension à la salle de lecture ${ }^{21}$.

Le rez-de-chaussée du bâtiment est occupé par les bureaux et la salle de lecture des archives municipales, dont les décors sont plus modestes.

A l'arrière, la partie en hémicycle est uniquement dédiée au stockage des collections. Cette forme est inhabituelle pour des magasins. L'architecte pensait qu'elle avait un double intérêt : protéger les livres en évitant de les exposer sous la lumière directe du jour ; permettre un gain de temps au magasinier en lui épargnant des allées et venues trop fastidieuses. Tous les étages étant bas de plafond, les livres sont à portée de main. La totalité des quatre étages est calculée pour conserver 400 ooo volumes. Cette disposition, conçue pour répondre à des besoins pratiques, a pourtant eu du mal à s'imposer : le Conseil supérieur des bâtiments civils, par exemple, se montre au début très sceptique ${ }^{22}$. Il faut toute la pédagogie de Sainsaulieu, de même que l'appui de Pol Neveux et d'Henri Loriquet ${ }^{23}$, pour réussir à imposer à tous les magasins rayonnants.

\section{Regards contemporains sur la bibliothèque}

" Aussitôt à l'intérieur, on est émerveillé ". Cette exclamation émaillant un article de La Construction moderne ${ }^{24}$ reflète assez bien les nombreux éloges écrits dans la presse juste après l'inauguration du bâtiment. Dès 1928, les journalistes apprécient " le luxe inouï ${ }^{25}$ " du nouveau temple de la lecture publique de Reims : ainsi Le Nord-Est vante sur plusieurs colonnes le " modernisme modéré dans lequel [l'architecte] a su [...] mener l'édifice ${ }^{26}{ }^{\prime}$.

Au-delà de la beauté du décor, nombreux sont ceux qui insistent sur la qualité des solutions pratiques offertes à l'utilisateur. Ainsi Forestier, dans La Construction moderne ${ }^{27}$, note que les problématiques de stockage, d'accroissement, de signalement et de communication des collections sont ici parfaitement résolues. Le plan rigoureux de Sainsaulieu s'affirme d'ailleurs comme un modèle à suivre : la bibliothèque municipale de Toulouse, construite quelques années plus tard par Jean Montariol (et dont le chantier a lui aussi été suivi par Pol Neveux), a su tirer profit de l'exemple rémois.

Il faut tout de même signaler que ce concert de louanges n'a pas été partagé, loin s'en faut, par le conservateur lui-même : Loriquet, dont les relations avec Sainsaulieu sont conflictuelles, ne cesse de pester dans sa correspondance contre certaines lacunes techniques (absence de lampes électriques dans les magasins, abandon du projet de fabrication d'un médaillier...) ; il critique également la débauche de faste déployée, l'importance démesurée de la surface du hall au détriment des salles d'accueil du public $^{28}$...

Il est vrai que ces espaces d'accueil, quoique très novateurs en 1928, ont finalement été victimes de leur succès. L'ancienne bibliothèque était très peu fré- 

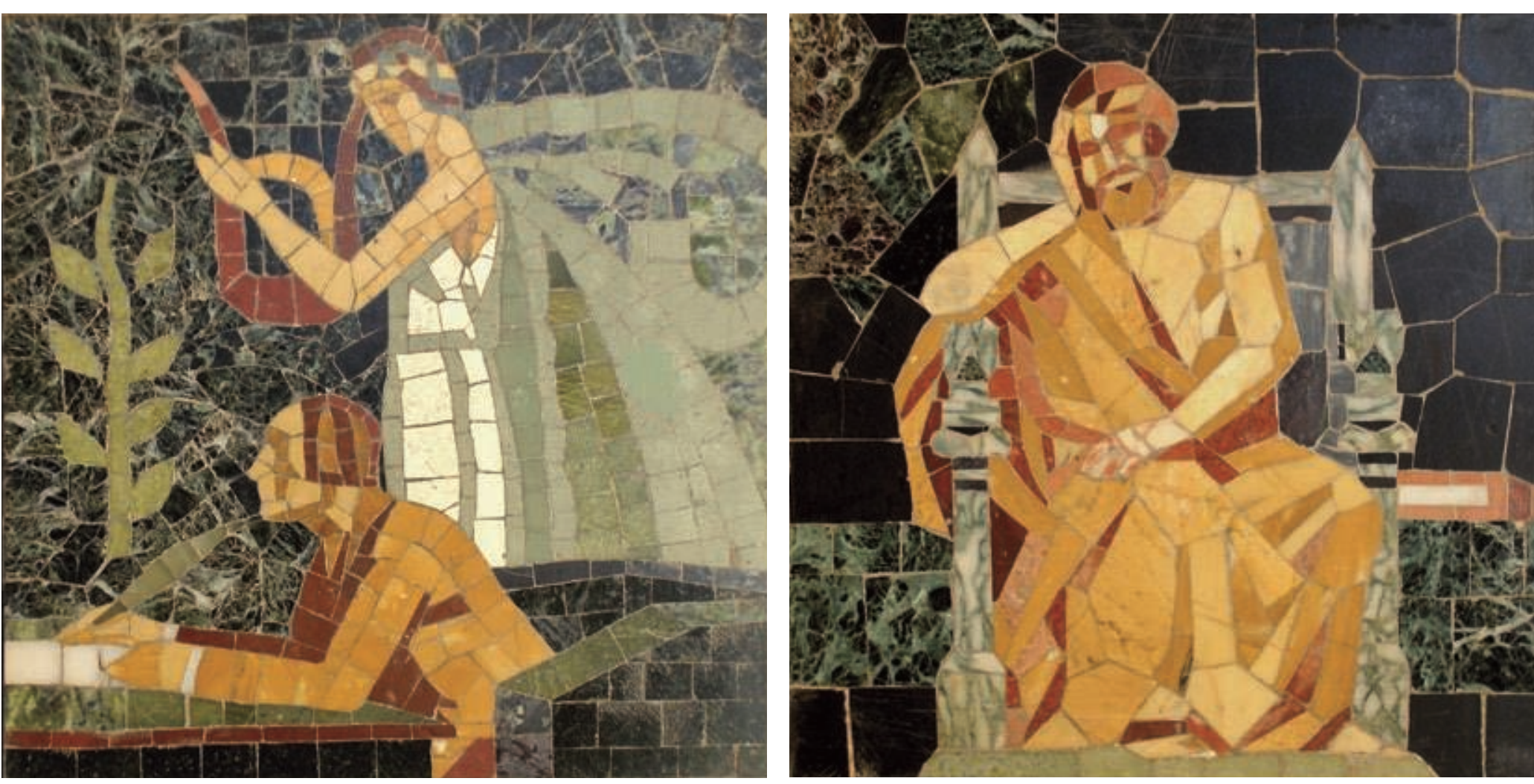

\La littérature (mosaïque du vestibule) $\quad$ La philosophie (mosaïque du vestibule) 


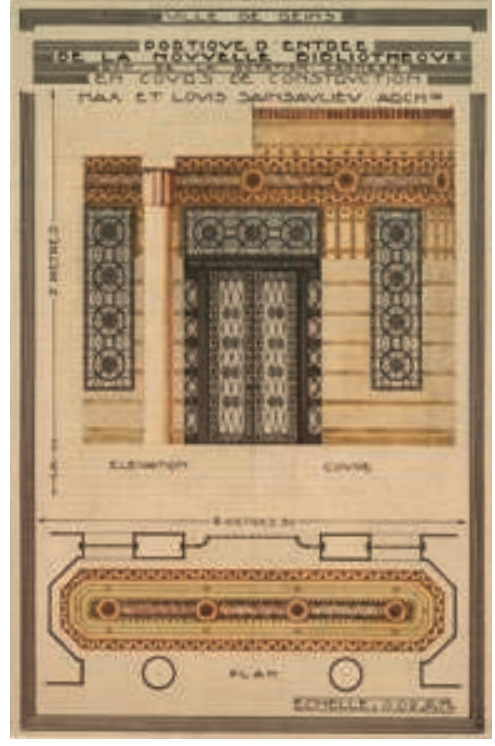

\Projet de Max Sainsaulieu pour une porte d'entrée

quentée : la bibliothèque Carnegie attire un public beaucoup plus nombreux ${ }^{29}$. A long terme, les salles de lecture se sont révélées étroites. Le manque de place a fini par devenir problématique, d'autant plus que de nouveaux usages et de nouveaux publics ont obligé à envisager des réajustements : comment accueillir les enfants ? Comment organiser l'accès aux périodiques, aux romans et à la littérature populaire lorsque toutes les collections sont stockées en magasin ? Ces questions, qui ont émergé dès la fin des années 1930, se sont posées avec une acuité plus grande après la Seconde Guerre mondiale, poussant Odette Réville, successeur de Loriquet, à écrire à propos de la bibliothèque : " A cette époque, une bibliothèque n'était pas encore considérée comme un organisme vivant, appelé à se développer en assurant constamment de nouvelles fonctions ; si certains services sont aujourd'hui logés dans des conditions défectueuses, la faute n'en saurait être imputée à ceux qui en avaient prévu les aménagements intérieurs il y a près de trente an $\mathrm{s}^{30}$ ». On lit dans cette note d'Odette Réville l'annonce des changements qui ont pu s'opérer en 2003-2005, avec la création d'une nouvelle médiathèque et la réhabilitation de la bibliothèque Carnegie comme lieu de conservation du patrimoine écrit.
Adoptant un schéma rationnel, idéalement située dans un quartier calme et proche de nombreuses institutions scolaires et universitaires, la bibliothèque Carnegie de Reims s'est construite autour d'une identité visuelle simple et forte : l'architecte a voulu en faire un nouveau temple du savoir ouvert sur la ville. Les emprunts à l'Antiquité, évoqués par le portique et son fronton majestueux, la symbolique encyclopédique et solennelle du grand vestibule, l'atmosphère propice au recueillement que dégage la salle de lecture, tous ces éléments se marient harmonieusement dans un ensemble sobre et géométrique, typique du style Art déco français des années 1920. La nouvelle bibliothèque veut s'inscrire dans la tradition littéraire locale, comme le rappellent les éléments décoratifs célébrant la ville de Reims et ses plus grands auteurs, mais elle invite aussi à s'ouvrir au monde, fidèle en cela à sa devise, qui est aussi celle de l'Académie nationale de Reims : " Educunt folia fructum " (les fleurs conduisent aux fruits).

\section{Matthieu Gerbault}

\section{Abréviations utilisées :}

$\mathrm{AD}:$ Archives départementales

AMC : Archives municipales et communautaires

BMVR : Bibliothèque municipale à vocation régionale 


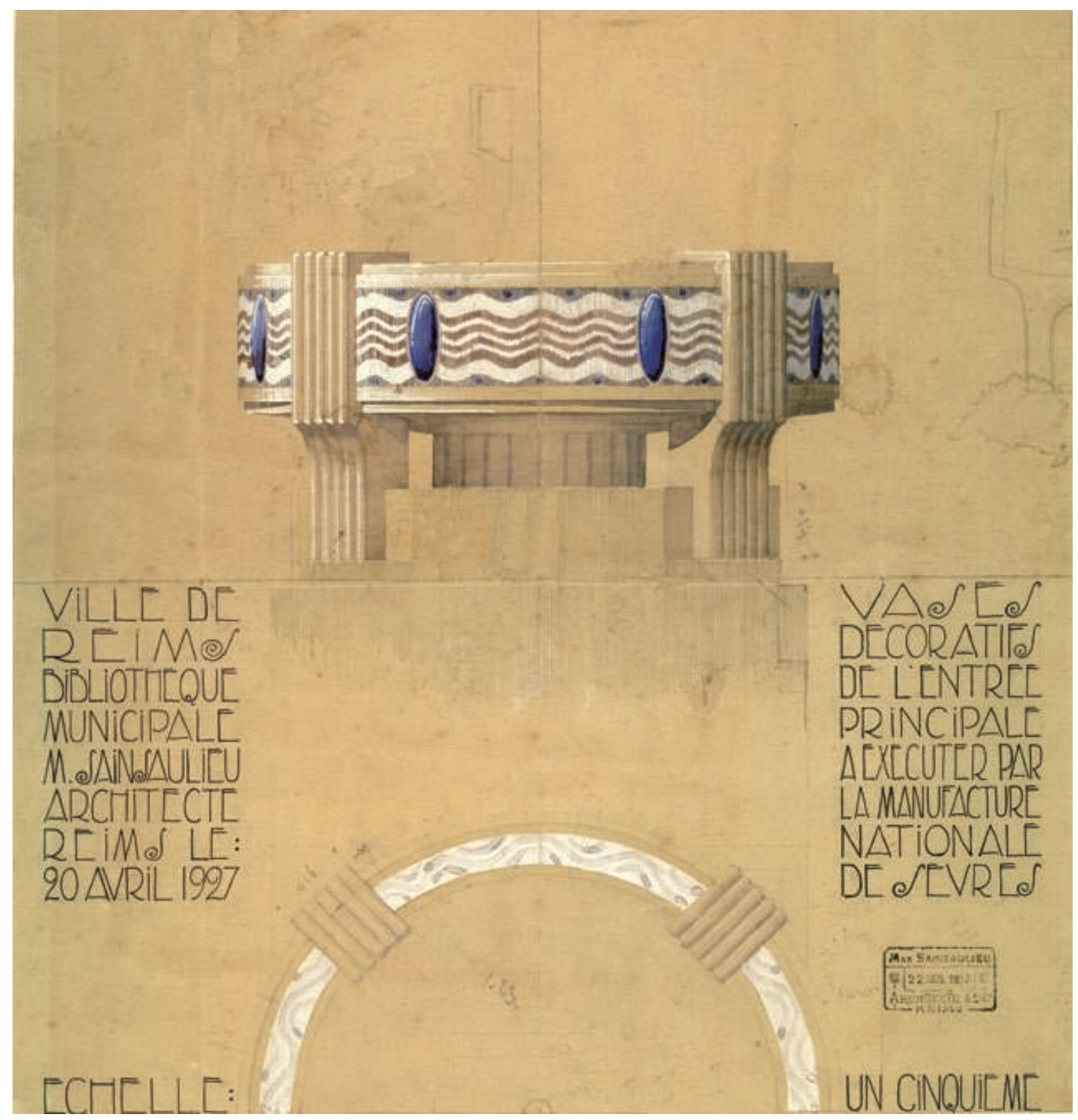




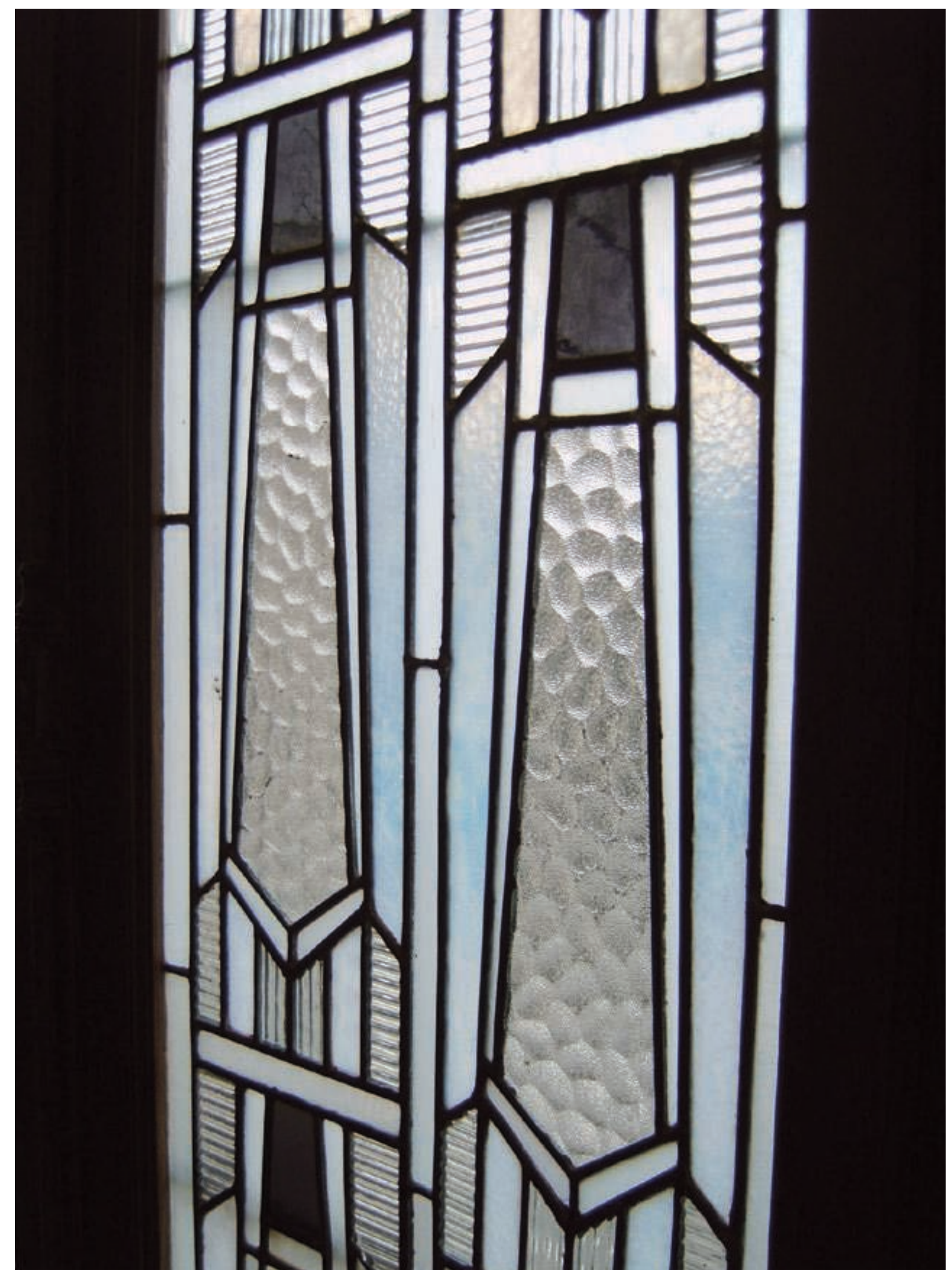

$\otimes$ Baie de la salle de lecture, par Jacques Gruber 


\section{Notes}

1 - Parmi l'abondante bibliographie publiée sur la bibliothèque à l'occasion de sa réouverture après travaux en 2005, on citera par exemple : Bibliothèque Carnegie - Reims : Concours 2006 " Les rubans du Patrimoine ". Reims : Ville de Reims, 2006.

2 - P. d'Estournelles de Constant, président du Centre européen de la dotation Carnegie. Lettre au maire de Reims, 25 novembre 1920. Reims, AMC, $31 \mathrm{~W} 65$.

3 - Lettre de Charles Roche, maire de Reims, à Max Sainsaulieu, 18 décembre 1920. Reims, AD Marne, 19 J 63 (Fonds Sainsaulieu).

4 - Sur Max Sainsaulieu et son œuvre, voir notamment «Le temps s'efface, l'œuvre demeure "-Max Sainsaulieu : un architecte rémois de la reconstruction [catalogue de l'exposition présentée à Saint-Julien les Villas, 2003, et à la Bibliothèque municipale de Reims, 2005], Reims : Ville de Reims, Châlons : Conseil général de la Marne, 2003.

5 - Voir sur ce point la correspondance entre Sainsaulieu et d'Estournelles de Constant, président du Centre européen de la dotation Carnegie, en 1923 (AD Marne, annexe de Reims, 19 J 63).

6 - Rapport sur le voyage d'étude effectué dans des bibliothèques françaises et suisses. 1er février 1921. Reims, AMC, 1 S 3 (Fonds Loriquet).

7 - Ibid., p. 1.

8 - Ibid., p. 2

9 - Examen du plan de la bibliothèque de Reims par la Commission supérieure des bibliothèques, réunie le 7 avril 1921 [en présence de Henri Omont, Pol Neveux, Alexandre Vidier, Henry Martin et Henri Loriquet], Reims, AMC, $1 \mathrm{~S}_{3}$.

10 - Reims, AMC, $31 \mathrm{~W} 65$

11 - Extrait de la délibération du conseil municipal du 6 avril 1922. Reims, AMC, $1 \mathrm{~S}_{3}$.

12 - Voir Reims, AMC, $1 \mathrm{~S}_{3}$.

13 - M. Chifflot, Rapport sur la construction d'une bibliothèque à Reims, 19 décembre 1922. AD Marne, annexe de Reims, 19 J 63 (Fonds Sainsaulieu).

14 - Ces réticences sont sobrement évoquées dans le courrier qui accompagne son projet modifié remis au Conseil supérieur des bâtiments civils et à la mairie de Reims le 16 mars 1923 (AD Marne, annexe de Reims, $19 \mathrm{~J} 63$ ). Elles s'étalent avec beaucoup plus d'emphase dans une lettre de Sainsaulieu à Pol Neveux datée du 21 janvier 1923 : fâché des demandes qu'il compare à des " ukases ", il accuse le Conseil supérieur des bâtiments civils de ne pas " respecter [sa] liberté d'artiste " et s'enorgueillit d'avoir " conçu une œuvre originale et peut-être même imprévue, mais à coup sûr très documentée et très mûrie, œuvre que [...] tous les professionnels de bibliothéconomie à qui nous l'avons soumise ont approuvée et même encouragée " (AD Marne, annexe de Reims, 19 J 63).

15 - AD Marne, annexe de Reims, $19 \mathrm{~J} 63$.

16 - Cette liste, proposée par l'Académie nationale de Reims, revue par Pol Neveux et approuvée par une commission municipale en 1925, est l'œuvre de Sediey (AD Marne, annexe de Reims, 19 J 65).

17 - AD Marne, annexe de Reims, 19 J 65.

18 - AD Marne, annexe de Reims, 19 J 65.

19 - Odette Réville. La Bibliothèque municipale de Reims, sa formation, son histoire, son état actuel. Reims : Matot-Braine, 1933, p. 21.

20 - AD Marne, annexe de Reims, $19 \mathrm{~J} 63$.

21 — « La salle dite des expositions a pour objet réel de servir à l'avenir d'agran- dissement à la salle de lecture, quand des temps meilleurs auront amené à la bibliothèque un nombre accru de lecteurs.

En attendant elle eût pu servir de salle de conférences ou de cours. Monsieur Loriquet a demandé, et finalement obtenu, qu'on en fasse une salle d'exposition. Je me souviens très bien que quand Monsieur Loriquet a proposé cela, Monsieur Pol Neveux a d'abord refusé net, disant qu'une bibliothèque n'est pas un musée et surtout que des livres précieux ne sont pas des objets d'exposition. " Lettre de M. Sainsaulieu à M. Valland, adjoint au maire, le 10 mai 1928 (AD Marne, annexe de Reims, 19 J 67).

22 - Voir sur ce point le rapport Chifflot, op. cit., du 19 décembre 1922 (Reims, AMC, $1 \mathrm{~S}_{3}$ ).

23 - Sur l'appui de Neveux et Loriquet, on se reportera à la correspondance qu'entretient Sainsaulieu avec eux (AD Marne, 19 J 63)

24 - Forestier, "Reims - La nouvelle bibliothèque municipale ", dans La Construction moderne, 9 septembre 1928, p. 589-594.

25 - Jean d'Héristal, " La nouvelle bibliothèque de Reims ", dans Le NordEst, 7 août 1928 .

26 - Ibid.

27 - Art. cit.

28 - Les griefs de Loriquet sont réunis dans une lettre qu'il adresse à Sainsaulieu le 28 novembre 1825 : «C'est vous et vous seul qui, de cœur léger et par amour de la gloriole, ne rêvant que de portique concours d'école, de hall de palace et de jardins superflus, n'avez même pas daigné venir conférer une seule fois avec moi des nécessités autrement sérieuses et puissantes que j'invoquais. Si je ne répugnais à troubler vos rêves, je pourrais vous donner l'amusante liste de sobriquets dont nos concitoyens qui me rencontrent ont baptisé et baptisent tous les jours votre œuvre " (AD Marne, $19 \mathrm{~J} \mathrm{63).} \mathrm{Loriquet} \mathrm{ajoute,} \mathrm{dans} \mathrm{une} \mathrm{lettre} \mathrm{à}$ Maurice Demaison datée du 2 mars 1928 : "Et dire que la presse des deux mondes, mal informée, célèbrera sur tous les tons la beauté architecturale, l'admirable adaptation technique de ma nouvelle maison, sans qu'il s'élève une voix pour en dire les tares. " (Reims, AMC, $\left.1 \mathrm{~S}_{3}\right)$.

29 - En 1923, la bibliothèque installée provisoirement au Musée des beauxarts n'attirait que 829 lecteurs, qui consultaient 1237 volumes; en 1930, la bibliothèque Carnegie accueille 11892 lecteurs pour 20098 communications.

30 - Odette Réville, "La Bibliothèque municipale de Reims ", dans La Champagne économique, 1950, p. 190-197. 